\title{
Gestão de Riscos na Indústria Farmacêutica
}

\section{Wolney Cardoso da Silva}

A gestão de riscos consiste no controle de ameaças e oportunidades, objetivando a sobrevivência, o alcance de vantagem competitiva sustentável e o sucesso das organizações, sendo desenvolvida por meio de processos sistemáticos integrados à estrutura, à cultura e às práticas organizacionais, contemplando todos os níveis organizacionais, processos, serviços e produtos. O porte, a complexidade, a importância econômica do setor e, especialmente, a elevada sensibilidade aos riscos têm exigido da indústria farmacêutica a melhoria da qualidade e aumento do rigor dos processos de gestão de riscos e sua extensão a todo o ciclo de vida de seus produtos. Este artigo objetiva a aplicação dos conceitos fundamentais da gestão de riscos no setor produtivo farmacêutico, com foco específico nos riscos relativos ao uso de medicamentos.

Palavras-chave: gestão de riscos; indústria farmacêutica.

The management of risks is characterized by control of threats and opportunities in order to survive, the scope of sustainable competitive advantage and success of organizations and has been developed through systematic processes to integrated structure, culture and organizational practices, including all levels organizational, processes, services and products. The size, complexity, the economic importance of the sector and especially the highly sensitive to the risks have required the pharmaceutical industry to improve quality and increase the accuracy of procedures for risk management and its extension to the whole life cycle of their products. This article aims to apply the basic concepts of risk management in the pharmaceutical manufacturing sector, with focus on specific risks related to the use of medicines.

Keywords: risk management; pharmaceutical industry. 


\section{Introdução}

O domínio do risco é fator decisivo à sobrevivência e ao sucesso do Homem e das organizações. Das intempéries, animais ferozes e luta pela conquista e manutenção de territórios, típicos dos momentos primitivos, até ao atual ambiente caracterizado pelo desenvolvimento tecnológico e condições de extrema competitividade e volatilidade, ocorreram mudanças notáveis nas características dos riscos e na forma com que o Homem e as organizações têm lidado com eles.

Ao longo da História, o Homem desenvolveu conhecimentos e técnicas para a abordagem racional de uma gama extensa e diversificada de fenômenos, permitindo a identificação, compreensão e tratamento das variáveis, condições e decisões necessárias ao controle das incertezas com as quais se depara. Vencidas as barreiras conceituais, foi possível o desenvolvendo de métodos e ferramentas sobre probabilidade, amostragem e estatística, bem como sobre o comportamento humano, propiciando as condições necessárias ao melhor tratamento dos riscos.

Apesar desse instrumental, o processo de tomada de decisão em condições de incerteza e a previsão do futuro, ainda se mantêm como desafios, uma vez que os padrões estabelecidos pela natureza garantem o retorno dos eventos em apenas parte dos casos, em razão da interdependência entre os eventos e da interferência humana; da não disponibilidade de informações em qualidade e em quantidade adequadas ä previsão sobre a ocorrência e o impacto dos eventos e do comportamento assimétrico do Homem frente à incerteza. Acomplexidade, e a grande variabilidade, tanto em freqüência quanto em amplitude, das condições ambientais das organizações, ampliam ainda mais as dimensões deste desafio, exigindo a disponibilidade de sistemas integrados de gestão, capazes de analisar adequadamente as demandas do ambiente interno e externo e atender as necessidades das diversas partes interessadas.

Modernamente, risco é conceituado como a probabilidade da ocorrência de um evento, condição ou circunstância, com potencial para provocar desvios, positivos ou negativos, nos resultados planejados ou esperados, sendo quantificado em função da combinação da probabilidade de ocorrência e da magnitude ou impacto de suas conseqüências. ${ }^{1}$ A gestão de riscos é o processo sistemático, de identificação, análise, implementação de respostas e monitoramento de um amplo espectro de variáveis, necessário ao controle de ameaças e de oportunidades, objetivando o alcance de vantagem competitiva sustentável, por meio da maximização das áreas nas quais se dispõe de algum controle sobre os resultados, e minimização daquelas nas quais este controle não é possível ou nas quais não se dispõe de informações para o estabelecimento da relação de causa e efeito. ${ }^{2}$ Além de sistemático o processo de gestão de riscos deve estar integrado à estrutura, à cultura e às práticas organizacionais, desenvolvendo-se em todos os níveis, processos e atividades da organização.

As organizações, como sistemas abertos, interagem com o ambiente específico de cada organização, caracterizado pelo mercado específico, fornecedores, concorrentes, e com o ambiente geral comum a todas as organizações. O processo de gestão de riscos deve contemplar as demandas e impactos relacionados com esse contexto externo, assim como com contexto interno da organização, e estabelecer mecanismos eficazes de comunicação com as diversas partes interessadas. ${ }^{3}$

Além da análise e definição dos contextos a ser considerados e o estabelecimento de mecanismos de comunicação com as partes interessadas, o processo de gestão contempla as atividades de estudo dos riscos, por meio da identificação, análise e avaliação; tratamento dos riscos; verificação ou monitoramento dos resultados e avaliação crítica e adequação. ${ }^{3}$ Com deve ser sistemático e contínuo, o processo de gestão de riscos enquadrase no modelo de controle de processos, denominado Ciclo PDCA, proposto por William Edwards Deming, ${ }^{4}$ conforme esquematizado na Figura 1.

A indústria farmacêutica é um setor complexo de grande importância econômica, com uma base científica e tecnológica relevante, que vem sofrendo rápidas e significativas mudanças estruturais, especialmente na busca de ganho de escala em produção e em pesquisa e desenvolvimento, cuja competitividade é fortemente influenciada pela capacidade de inovação tecnológica contínua. ${ }^{5} \mathrm{O}$ processo de produção do setor é multidisciplinar, envolvendo em sua cadeia produtiva uma variada e extensa gama de fornecedores, prestadores de serviços e profissionais de diversas 
áreas de conhecimento, ${ }^{6}$ comportando quatro estágios de desenvolvimento tecnológico: pesquisa e desenvolvimento; produção de farmoquímicos; produção de especialidades farmacêuticas; marketing e comercialização de especialidades farmacêuticas. ${ }^{6}$

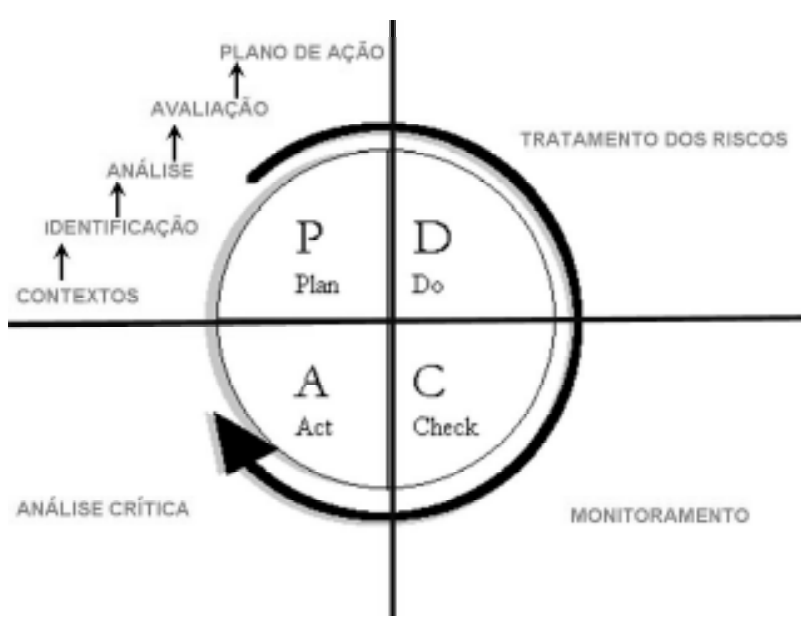

Figura 1: Processo de gestão de riscos modelado de acordo com o Ciclo PDCA

Pesquisas realizadas nos Estados Unidos da América ${ }^{7}$ demonstram que o setor produtivo farmacêutico apresenta riscos significativos com relação a outros segmentos econômicos e que é altamente sensível aos impactos de eventos positivos ou negativos. Apesar da predominância de cultura de controles, o setor pode não alcançou o nível de excelência praticado por outros segmentos, como o financeiro e de seguro, apresentando o seguinte panorama:

i. Predominam o processo subjetivo e a postura reativa na abordagem dos riscos;

ii. Não há padronização do processo de gestão de riscos;

iii. Está ocorrendo conscientização quanto à necessidade de adequação organizacional para a gestão de riscos, evidenciada pelo aumento da freqüência de referências aos riscos nos relatórios das empresas;

iv. Os riscos mais frequentemente relatados estão relacionados com as mudanças no ambiente competitivo, retenção de talentos, produtos em desenvolvimento, atraso no lançamento de produtos e suprimento de insumos;

v. As empresas com pior desempenho na gestão de riscos são as dotadas de estruturas organizacionais estanques e as de melhor desempenho são que adotam a estrutura matricial para a gestão de riscos.

\section{Aplicação do processo de gestão de riscos}

Em função do porte, complexidade e características da organização, o processo de gestão demanda a aglutinação dos riscos em dimensões específicas, de forma a proporcionar uma visão estratégica consolidada, 8 sugerindo-se para o setor farmacêutico as seguintes dimensões de risco: mercado, produto, operacional e legal. As mudanças no ambiente competitivo, evidenciado como o principal risco, ${ }^{7}$ a capacidade de inovação e a capacidade de introdução contínua de novos medicamentos no mercado, apontados como diferencial competitivo e principal fator de sucesso, ${ }^{9,10}$ estão inseridas nas quatro dimensões de riscos sugeridas. ${ }^{6}$

O setor farmacêutico vem sofrendo pressões no sentido da melhoria da qualidade científica das decisões sobre a relação entre os benefícios e os riscos de seus produtos, ${ }^{11}$ contemplando a gestão de riscos durante todo o ciclo de vida do produto, desde os testes pré-clínicos e clínicos até a fase de pós-comercialização, característica das atividades de farmacovigilância. ${ }^{12}$ Em razão dessas pressões; da notoriedade que proporciona; por envolver as quatro dimensões de riscos sugeridas; da velocidade de produção de novas tecnologias, excipientes, formulações e indicações; dos impactos financeiros e sobre a imagem das empresas e de seus dirigentes; do aumento do número de consumidores e a possibilidade de impactos individuais e à saúde pública, o risco de reações adversas a medicamentos (RAM) foi selecionada para a contextualização do processo de gestão de riscos na indústria farmacêutica.

\section{DEFINIÇÃO DO CONTEXTO EXTERNO}

O ambiente externo é composto por consumidores; profissionais e empresas da área de saúde; distribuidores e revendedores; órgãos nacionais e internacionais de monitoramento de problemas relacionados ao uso de medicamentos; organizações de defesa do 
consumidor; órgãos de regulação, controle e fiscalização; controladores, acionistas e financiadores. $\mathrm{O}$ ambiente externo tem como principal característica a pressão social e regulamentar no sentido de que as empresas assumam a responsabilidade sobre os efeitos de seus medicamentos durante todo o ciclo de vida dos produtos, monitorando, tratando e informando as autoridades e consumidores sobre os eventuais problemas relacionados ao uso de medicamentos.

Em decorrência da gradual conscientização quanto aos direitos do consumidor e do estabelecimento de dispositivos legais de proteção, os problemas relacionados ao uso de medicamentos representam ameaças significativas à imagem, aos resultados e à própria sobrevivência das empresas, em decorrência, em especial, dos impactos financeiros e à imagem. A atitude pró-ativa com relação ao risco pode, por outro lado, se caracterizar em oportunidades para melhoria da imagem junto à sociedade, consumidores e órgãos de regulação e controle e pela possibilidade de identificação de novas indicações para o produto.

\section{DEFINIÇÃO DO CONTEXTO INTERNO}

Internamente, a alta direção e os componentes de gestão de riscos organizacionais, de pesquisa e desenvolvimento, de produção e de qualidade estão envolvidos na gestão do risco relacionado ao uso de medicamento. É interessante a constituição de comitê específico para a gestão desse risco, constituído por representantes das principais áreas envolvidas.

\section{DEFINIÇÃO DO CONTEXTO INTERNO DA GESTÃO DO RISCO}

Agestão de riscos associados ao uso de medicamentos está inserida do contexto da farmacovigilância, caracterizada pelo conjunto de atividades de identificação precoce, avaliação, compreensão e prevenção de efeitos adversos ou relacionados ao uso, após a introdução do medicamento no mercado, objetivando a promoção do uso seguro de medicamentos pela população. ${ }^{13}$

Em razão de sua importância estratégica, a gestão desse risco será contemplada na política geral da organização, garantindo-se os recursos necessários à sua consecução e definindo-se as atribuições e responsabilidades das partes envolvidas. Os objetivos da gestão do risco são a identificação de falhas nos processos de pesquisa e desenvolvimento, comercialização e marketing; a redução das taxas de morbidade e de mortalidade; redução do impacto financeiro e sobre a imagem da empresa; comunicação aos órgãos de regulação, fiscalização e controle, profissionais da área de saúde e consumidores em geral e a identificação de eventuais oportunidades de melhoria do posicionamento competitivo.

\section{DEFINIÇÃO DOS CRITÉRIOS DE RISCO}

$\mathrm{O}$ estudo e tratamento dos riscos relacionados ao uso de medicamentos têm como principal critério de abordagem o consumidor final, tanto individual como coletivamente, contemplando análises do ponto de vista de marketing, técnico, financeiro, legal, humanitário, social. No âmbito deste artigo, serão considerados apenas os critérios quanto ao consumidor como paciente.

\section{COMUNICAÇÃO COM AS PARTES INTERESSADAS}

O ciclo de comunicação e consulta com relação aos riscos associados ao uso de medicamentos é constituído por pacientes; profissionais e organizações da área de saúde; pesquisadores da academia e do setor produtivo; indústria farmacêutica; revendedores de medicamentos; órgãos de regulação, fiscalização e controle e organizações e sistemas de farmacovigilância. Os instrumentos de comunicação são as notificações de Reações Adversas de Medicamento (RAM), alertas e informes das organizações de farmacovigilância, bulas, prospectos, notas, artigos técnicos e científicos, informativos e matérias na imprensa comum ou especializada, difundidos em meio eletrônico ou impresso, ou em congressos, seminários, palestras e cursos específicos. ${ }^{14}$

\section{IDENTIFICAÇÃO DOS RISCOS}

Consiste na identificação de problemas de segurança não identificados antes da comercialização do medicamento, por meio da identificação de casos clínicos individuais ou de séries históricas. ${ }^{13} \mathrm{O}$ principal risco relacionado ao uso de medicamentos é caracterizado pela Reação Adversa ao Medicamento (RAM), ${ }^{15}$ definida como "reação nociva e involuntária, que ocorre em doses geralmente utilizadas no homem para 
profilaxia, diagnóstico, ou tratamento de doença ou para a modificação de funções fisiológicas". ${ }^{16}$ Atualmente, além da RAM, foram agregados ao contexto da gestão do risco: $13,15,16$

i. Perda de eficácia;

ii. Desvios de qualidade;

iii. Uso indevido acidental ou deliberado;

iv. Abuso;

v. Intoxicação;

vi. Falha terapêutica; vii. Erros de medicação;

viii. Evento adverso;

ix. Interações medicamentosas;

x Efeitos colaterais positivos ou negativos.

As reações adversas a medicamentos (RAM) podem ser classificadas conforme indicado na Figura 2, evidenciando que as RAM do tipo "B", não relacionadas à dose, são mais difíceis de predizer e de identificar.

\begin{tabular}{|c|c|c|c|}
\hline Tipo de reação & Mnemônico & Características & Exemplos \\
\hline $\begin{array}{c}\mathrm{A} \\
\text { Relacionada à dose }\end{array}$ & Aumento & $\begin{array}{l}\text { - } \text { Comum } \\
\text { - Relacionada a um efeito farmacológico da droga } \\
\text { - Esperada } \\
\text { - Baixa mortilidade }\end{array}$ & $\begin{array}{l}\text { - Efeitos tóxicos: Intoxicação; síndrome } \\
\text { serotoninérgica com ISRSs } \\
\text { - Efeitos colaterais: efeitos } \\
\text { anticolinérgicos de antidepressivos } \\
\text { tricíclicow }\end{array}$ \\
\hline $\begin{array}{l}\mathrm{B} \\
\text { Não relacionada à } \\
\text { dose }\end{array}$ & Bizarro & $\begin{array}{l}\text { - Incomum } \\
\text { - Não relacionada a um efeito farmacológico da droga } \\
\text { - Inesperada } \\
\text { - Alta mortilidade }\end{array}$ & $\begin{array}{l}\text { - Reações imunológicas: } \\
\text { hipersensibilidade à penicilina } \\
\text { - Reações idiossincráticas: porfíria aguda, } \\
\text { hipertermia maligna, pseudoalergia (ex: } \\
\text { rash em uso de amplicilina) }\end{array}$ \\
\hline $\begin{array}{l}\text { C } \\
\text { Relacionada à dose e } \\
\text { ao tempo de uso }\end{array}$ & Crônico & $\begin{array}{l}\text { - Incomum } \\
\text { - Relacionado ao efeito cumulativo do fármaco }\end{array}$ & $\begin{array}{l}\text { - Supressão do eixo hipotalâmico - } \\
\text { hipofisário - adrenal por corticosteróides }\end{array}$ \\
\hline $\begin{array}{l}\text { D } \\
\text { Relacionada ao temo } \\
\text { de uso }\end{array}$ & Atraso & $\begin{array}{l}\text { - Incomum } \\
\text { - Normalmente relacionado à dose } \\
\text { - Ocorre ou aparece algum tempo após o uso do } \\
\text { medicamento }\end{array}$ & $\begin{array}{l}\text { - Teratogênese (ex.: adenocarcionoma } \\
\text { associado ao dietiletilbestrol) } \\
\text { - Carciongênese } \\
\text { - Discinesia tardia }\end{array}$ \\
\hline $\begin{array}{c}\text { E } \\
\text { Abstinência }\end{array}$ & Fim do uso & $\begin{array}{l}\text { - Incomum } \\
\text { - Ocorre logo após a suspensão do medicamento }\end{array}$ & $\begin{array}{l}\text { - Síndrome da abstinência a opiáceos } \\
\text { - Isquemia miorcárdica (supressão de } \\
\text { â-bloqueador) }\end{array}$ \\
\hline $\begin{array}{l}\text { F } \\
\text { Falha esperada na } \\
\text { terapia }\end{array}$ & Falha & $\begin{array}{l}\text { - Comum } \\
\text { - Relacionada à dose } \\
\text { - Frequentemente causado por interação de } \\
\text { medicamentos }\end{array}$ & $\begin{array}{l}\text { Dosagem inadequada de } \\
\text { anticoncepcional orar, particularmete } \\
\text { quando utilizados indutores enzimáticos }\end{array}$ \\
\hline
\end{tabular}

Figura 2: Classificação de reações adversas a medicamentos - continuação. ${ }^{15}$ 


\section{ANÁLISE DOS RISCOS}

A análise dos riscos consiste na caracterização do nível de risco (NR) de cada evento, constituído pela combinação da probabilidade de ocorrência e da extensão de seu impacto. No caso das RAM's, devem ser avaliadas a força da relação de casualidade e a freqüência dos eventos clínicos identificados. Para a análise dos riscos, o UPPSALA MONITORING CENTRE (UMC) prevê os seguintes protocolos para análise de riscos de RAM: ${ }^{16}$

\section{i. Avaliação da causalidade de eventos com RAM}

- Certo: um evento clínico que ocorre em um prazo plausível após a administração do medicamento e que não pode ser explicado por doença concomitante ou outros medicamentos. A reação à retirada do medicamento deve ser clinicamente plausível. $\mathrm{O}$ evento deve ser farmacologicamente definitivo, executando-se, se necessário, procedimento de re-exposição;

- Provável: um evento clínico que ocorre em um prazo razoável após administração do medicamento, com baixa possibilidade de ser atribuído a doença concomitante ou a outro medicamento ou substâncias químicas e que oferece uma resposta clínica razoável à retirada do medicamento. Não é requerida a reexposição;

- Possível: um evento clínico que ocorre em um prazo razoável após administração do medicamento, mas que pode ser explicado por doença concomitante ou outros medicamentos ou substâncias químicas. Não se requer informação sobre o efeito da retirada da droga;

- Improvável: um evento clínico no qual o tempo decorrido entre a administração do medicamento e os efeitos investigados não indica uma relação de casualidade e em que outras drogas, produtos químicos ou doença subjacente fornecem explicações plausíveis;

- Condicional: mm evento clínico que requer dados adicionais ou análise mais detalhada dos dados disponíveis;

- Não classificável: um evento clínico que não pode ser avaliado, em decorrência da insuficiência ou inconsistência das informações disponíveis.

ii. Para a probabilidade a UMC recomenda a adoção do critério esquematizado na Figura 3, que representa a freqüência de casos na população estudada;

iii. Quanto ao impacto ou conseqüência a UMC estabelece os seguintes critérios para as reações adversas que devem ser consideradas como "graves": 16

- resultar em incapacidade significativa ou persistente;

- requerer hospitalização ou o dilatação do tempo de hospitalização do paciente;

- oferecer risco à vida do paciente;

- resultar em morte.

iv. São também consideradas como reações graves a malformação congênita e os efeitos clinicamente significantes; ${ }^{14,15}$

vi. As reações não enquadradas nas condições acima relatadas são consideradas "não graves". ${ }^{15}$

\begin{tabular}{|c|l|l|}
\hline \multirow{2}{*}{ Classificação } & \multicolumn{2}{|c|}{ Freqüência } \\
\cline { 2 - 3 } & \multicolumn{1}{|c|}{ ocorrência/população } & \% \\
\hline Muito comum & $>1 / 10$ & $>10$ \\
\hline $\begin{array}{c}\text { Comum } \\
\text { (freqüente) }\end{array}$ & $>1 / 100 \mathrm{e}<1 / 10$ & $>1 \mathrm{e}<10$ \\
\hline $\begin{array}{c}\text { Incomum } \\
\text { (infrequente) }\end{array}$ & $>1 / 1.000 \mathrm{e}<1 / 100$ & $>0,1 \mathrm{e}<1$ \\
\hline Rara & $>1 / 10.000 \mathrm{e}<1 / 1.000$ & $>0,01 \mathrm{e}<0,1$ \\
\hline Muito rara & $>1 / 10.000$ & $>0,01$ \\
\hline
\end{tabular}

Figura 3: Classificação das reações adversas a medicamento quanto à freqüência. ${ }^{16}$

\section{AVALIAÇÃO DOS RISCOS}

O nível de risco obtido da composição entre a relação de casualidade, a freqüência e a gravidade das conseqüências dos eventos observados será o parâmetro utilizado para a categorização e avaliação dos riscos, tendo como principal objetivo a revisão periódica da relação entre os benefícios e os riscos oferecidos pelo medicamento sob análise. ${ }^{13,14}$ A relação benefícios/riscos deve ser avaliada em variados aspectos de uma perspectiva dos interesses da empresa, tais como financeiro, de imagem, legal, cultural, de mercado, operacional. A análise da relação benefícios/riscos também deve ser realizada de uma perspectiva social e econômica e da aceitabilidade social. ${ }^{13,15}$

\section{TRATAMENTO DOS RISCOS}

A partir das avaliações da relação benefícios/riscos são selecionadas as diversas opções de tratamento, objetivando a redução da freqüência e da gravidade das RAM's com impactos negativos e a exploração 
das eventuais oportunidades daquelas com resultados positivos. A natureza, extensão e profundidade das medidas de tratamento dos riscos podem variar em uma larga faixa de opções, em função das características da organização, do mercado, da sociedade e de cada risco em particular, podendo ser apontadas as seguintes alternativas genéricas, relativas à natureza do medicamento e das condições de uso: ${ }^{13,14,15}$

i. Comunicação dos riscos aos órgãos públicos de regulação e fiscalização e organizações especializadas em monitoramento de RAM's;

ii. Investigação das estratégias, procedimentos e recomendações quanto ao uso adequado e quanto à prevenção de RAM's;

iii. comunicação dos riscos e recomendações aos profissionais de saúde e pacientes;

iv. Alteração de textos de bulas, prospectos e informativos distribuídos aos usuários e profissionais da área de saúde;

v. Qualificação de médicos e outros profissionais da área de saúde;

vi. Adequação dos procedimentos de monitoramento da qualidade de insumos, produtos, instalações e utilidades;

vii. Aperfeiçoamento dos procedimentos de desenvolvimento de medicamentos;

viii.Adequação do produto;

ix. Lançamento de novos produtos;

x. Retirada do produto do mercado.

\section{MONITORAMENTO E ANÁLISE CRÍTICA}

A natureza, freqüência e gravidade das RAM's devem ser monitoradas e analisados de forma permanente, para avaliação da efetividade, eficiência e eficácia dos tratamentos aplicados ao risco, bem como para identificação de eventuais mudanças nas condições que possam afetar aqueles fatores, os custos dos tratamentos e os resultados esperados. Eventuais adequações que se mostrarem necessárias devem ser planejadas e implantadas, repetindo-se o ciclo tantas vezes quantas se fizerem necessárias.

\section{Conclusões}

Em decorrência do ambiente competitivo e turbulento e de novas demandas sociais, econômicas e legais, além do cumprimento da missão e do alcance de metas específicas, as organizações têm sido cada vez mais exigidas no sentido de demonstrar a disponibilidade de sistemas estruturados de gestão de riscos. No caso da indústria farmacêutica, a implementação de processos sistemáticos de gestão de riscos é ainda mais premente, em razão de sua alta sensibilidade, do impacto decorrente da emergência de novas tecnologias e do processo de reestruturação do setor.

\section{REFERÊNCIAS}

1. FERMA - Federation of European Risk Management Associations. Estándares de Gerencia de Riesgos. Bruxelas, 2003. Disponível em $<$ http://www.theirm.org/publications/documents/ rm_standard_spanish_15_11_04.pdf $>$ Acessado em $14 / 03 / 2008$

2. Bernstein, Peter 1. Desafio aos Deuses: A Fascinante História do Risco. Tradução de Ivo Korylowski 18. ed. Rio de Janeiro: Elsevier, 1997.

3. Comitê OB - 007 de Gestão de Riscos, Standards Austrália e Standard New Zealand. Gestão de Riscos - A Norma AS/NZS 4360:2004. Risk Tecnologia Editora. São Paulo, 2004.

4. Falconi, Vicente Campos. Qualidade Total: Padronização de empresas. Belo Horizonte: Fundação Cristiano Otoni, 1992.

5. Andricopulo, Adriano D.; Garrat, Richard D.; OLIVA, Glaucius. Revista Estudos. Goiânia, v.32, n. 11, nov. 2005.

6. Vieira, Vera M. da Motta, Ohayon, Pierre. Inovação em Fármacos e medicamentos: Estado-da-arte o Brasil e políticas de P\&D. 2006. Disponível em $<$ hhttp://www.pucmg.br $>$. Aecessado em 16/05/2008.

7. KPMG. Pharmaceutical companies need to improve their risk management framework: KPMG. Continuity Central, 2005. Disponível em $<$ http:// www.continuitycentral.com $>$. Acessado em 20/05/2008. 
8. Duarte Júnior, Antonio Marcos; Pinheiro, Fernando Antonio Perrone; JORDÃO, Manoel Rodrigues; BASTOS, Norton Torres. Gerenciamento de riscos corporativos: Classificação, definições e exemplos. UNIBANCO Global Risk Management, São Paulo, 2000.

9. Alvarez, Vitor M. Pelaez. G.D. Searle: Os limites de crescimento de uma empresa Farmacêutica. 1998. Textos para Discussão. Disponível em: <http://www. economia.ufpr.br/publica/textos/1998/txt0298\%20 Victor\%20limite\%20cresc.doc $>$ Acessado em $12 / 05 / 2008$

10. Hermel, Philippe, Bertoli, Annie. The TQM Magazine, v. 13, n. 3, 2001.

11. Edward, Brian. Drug Safety 2004, 27(08):609-617.

12. Tsintis, Panos; La Mache, Edith. Drug Safety 2004, 27(8):509-517.

13. Dias, Murilo Freitas; Souza, Nair R.; Bittencourt, Milena O.; Nogueira, Márcia S. Fármacos \& Medicamentos, São Paulo, 2005, v.34, n. VI.
14. Dias, Murilo Freitas. Fármacos \& Medicamentos, São Paulo, 2004, v. 31, n. V.

15. Figueiredo, Patrícia Mandali de; Costa, Alessandra A.; Santa Cruz, Fernanda do C.; Melo, José R. R.; Nogueira, Márcia S.; Góes, Tâmara P. A. Fármacos \& Medicamentos, São Paulo, 2005, v. 34, n. VI.

16. UMC, The Uppsala Monitoring Centre. Practical Pharmacovigilance. Disponível em $<$ http://www. who-umc.org > Acessado em 03/06/2008.

\section{Wolney Cardoso da Silva ${ }^{* 1,2}$}

${ }^{1}$ Analista Técnico do Banco Central do Brasil

${ }^{2}$ Faculdade de Tecnologia SENAI Roberto Mange, Anápolis, Brasil

E-mail: wolney.cardosodasilva@gmail.com.br 